\title{
Distinguishing CP-odd couplings of the Higgs boson to weak boson pairs
}

\author{
Siddharth Dwivedi ${ }^{1 *}$, Dilip Kumar Ghosh ${ }^{2 \dagger}$, Biswarup Mukhopadhyaya ${ }^{1 \ddagger}$ and Ambresh \\ Shivaji ${ }^{3 \S}$ \\ ${ }^{1}$ Regional Centre for Accelerator-based Particle Physics, Harish-Chandra Research \\ Institute, Chhatnag Road, Jhunsi, \\ Allahabad - 211019, India \\ ${ }^{2}$ Department of Theoretical Physics, \\ Indian Association for the Cultivation of Science, \\ $2 A \& 3$ 2B Raja S.C. Mullick Road, \\ Kolkata - 700032, India \\ ${ }^{3}$ INFN, Sezione di Pavia, \\ Via A. Bassi 6, 27100 Pavia, Italy
}

August 29, 2018

\begin{abstract}
We consider the observable effects of $\mathrm{CP}$-violating anomalous $Z Z h$ interaction arising from gauge invariant dimension-6 operators at the Large Hadron Collider (LHC), with the purpose of distinguishing them from not only the standard model effects but also those of CP-even anomalous interactions of similar nature. The postulation of a gauge invariant origin makes various couplings of this kind interrelated. The updated constraints from the LHC as well as limits from neutron and electron dipole moments are used in selecting the benchmark interaction strengths. We use some asymmetry parameters that have no contribution from standard or $\mathrm{CP}$-even anomalous interactions. Parton showering and detector level simulation is included to make our analysis as realistic as possible. On the whole, we conclude that gauge invariant interaction of strength $\geq 40 / \mathrm{TeV}^{2}$ can be successfully isolated using integrated luminosities in the $1.5-3.0 \mathrm{ab}^{-1}$ range.
\end{abstract}

\section{Introduction}

As results from the high energy run of the Large Hadron Collider (LHC) start arriving, interest in probing any non-standard behavior of the scalar with mass around 125-126 GeV [1,2] remains ever-alive. To keep the flame on, departure from the pattern of its interactions as laid down in the standard model (SM) is still allowed, though not imperatively, by the $(7+8) \mathrm{TeV}$ data. On

\footnotetext{
${ }^{*}$ E-mail: siddharthdwivedi@hri.res.in

${ }^{\dagger}$ E-mail: tpdkg@iacs.res.in

${ }^{\ddagger}$ E-mail: biswarup@hri.res.in

${ }^{\S}$ E-mail: ambresh.shivaji@pv.infn.it
} 
the other hand, issues ranging from the naturalness of the Higgs mass to the question of vacuum stability continue to nudge theorists even after Higgs discovery. Therefore, it is both legitimate and well-motivated to enquire if the high energy run can reveal some non-standard interactions of the particle which, in all fairness, can be called 'a Higgs boson'. Some such investigations in relation to $\mathrm{CP}$-violating $Z Z h$ coupling are reported here.

A rather general approach is to consider effective interactions of the Higgs, without committing oneself to any specific theoretical model. In this spirit, many studies have taken place over the years, probing higher-dimensional $W W h$ and $Z Z h$ interactions, often taking them in isolation and predicting observable phenomena in terms of the new operators [3 56]. It is, however, worthwhile to obtain these interactions in terms of gauge invariant higher-dimensional operators [57 62]. This is primarily because these operators can arise, if at all, by integrating out hitherto unseen new physics, and such physics, ruling above the electroweak symmetry breaking scale, must by default be $S U(2)_{L} \times U(1)_{Y}$ invariant. Also, the $W W h, Z Z h, \gamma \gamma h$ and $\gamma Z h$ interaction terms ensuing from such gauge invariant operators are correlated, and this correlation percolates into observed phenomena involving Higgs production as well as decay. A detailed inventory as well as a large mass of work already exists on CP-even operators of this kind and their constraints and consequences. Systematic studies on their CP-odd counterparts are relatively sparse, and perhaps less exhaustive. However, the very observation of the baryon asymmetry in the universe strongly suggests some source of CP-violation over and above what is manifested in the Cabibbo-Kobayashi-Maskawa scheme of the SM. A bottom-up investigation of CP-odd couplings of the recently discovered scalar is therefore as well-founded as one on their CP-even counterparts.

In general, there are five each of CP-even and-odd operators of dimension-6 involving $V V h$ interactions. Coefficients of the latter are subject to rather strong constraints from the limits on the electric dipole moments (EDM) of the neutron and the electron and also from the upper bounds on $\mathrm{Hg}$ EDM [63,64], in addition to global fits of collider data (including those from the LHC) that constrain the CP-even operators as well. Arguably a detailed study of these constraints on the $\mathrm{CP}$-violating operators was presented in the reference [65. It is, however, possible to generalize the study even further. First, the constraints from EDM are rather tight on individual operators which tend to get very strongly correlated if there are two or three of them present at a time. On the other hand, taking them together along with other dimension- 6 operators constructed out of the fermion fields and the Higgs doublet [57,58], which can potentially contribute to fermion EDMs, can relax such correlations considerably, and one can end up with large values of the effective $V V h$ couplings that potentially contribute to collider observables. Moreover, once we introduce the gauge invariant effective interaction terms, the simultaneous existence of several of them is the general expectation, unless some specific symmetries are invoked to forbid some of them. Secondly, global fits of collider data can yield less stringent constraints if CP-odd and-even operators are all present simultaneously, mainly due the sheer proliferation of parameters (which nonetheless should be there in the general effective theory). On the other hand, it is only natural to expect operators of both kinds of a given dimensionality to exist, in the absence of any forbidding principle.

To further elaborate on the second issue mentioned above, we need to identify some observables, mostly defined in terms of asymmetries, which subtract out the effect of CP-even operators, while keeping substantial separation between the CP-odd ones with the standard model predictions including their uncertainties. This is accomplished in the present work, allowing in principle the existence of all CP-odd and-even gauge invariant dimension- 6 operators. The points we emphasize in particular are as follows:

- Constraints from collider as well as EDM limits are listed in terms of the effective $V V h$ couplings, though they can arise from all possible operators at the same time. 
- The contributions of the CP-even operators to the suggested asymmetries at the LHC lie within the $1 \sigma$ error-bars of the SM, while the CP-odd ones are shown to lead to values that steer clear of these error-bars over extended regions of the parameter space.

- The prediction of observable asymmetries is based on detailed event generation, including showering, jet formation and detector simulation, thus leading to realistic estimates of what can be observed.

- Our 'realistic' predictions of CP-violating asymmetries and suppression of the effects of CPeven operators are presented for various luminosities, giving an idea of the luminosity required to obtain useful conclusions.

- Correlated interactions arising due to the gauge-invariant nature of the parent operators are always taken into account, taking, for example, the $\gamma Z h$ and $\gamma \gamma h$ couplings arising concomitantly from the dimension-6 operators (and thus competing favorably with their loop-induced SM counterparts).

A resume of the CP-odd dimension- 6 operators as well as their current limits is given in section 2. In section 3 we discuss the observables which elicit the presence of $\mathrm{CP}$-violating operators and help distinguish them from the SM and the corresponding CP-even counterparts. The role of asymmetries constructed from the kinematic distributions of such observables is also discussed. We summarize and conclude in section 4 .

\section{CP-odd anomalous couplings and their constraints}

The set of CP-violating gauge-Higgs operators of dimension- 6 that we have considered in our analysis [65] is listed below :

$$
\begin{aligned}
\tilde{O}_{W} & =\frac{\tilde{f}_{W}}{\Lambda^{2}}\left(D_{\mu} \Phi\right)^{\dagger} \hat{\tilde{W}}^{\mu \nu}\left(D_{\nu} \Phi\right) ; \quad \tilde{O}_{B}=\frac{\tilde{f}_{B}}{\Lambda^{2}}\left(D_{\mu} \Phi\right)^{\dagger} \hat{\tilde{B}}^{\mu \nu}\left(D_{\nu} \Phi\right) ; \\
\tilde{O}_{B B} & =\frac{\tilde{f}_{B B}}{\Lambda^{2}} \Phi^{\dagger} \hat{B}^{\mu \nu} \hat{\tilde{B}}_{\mu \nu} \Phi ; \quad \tilde{O}_{W W}=\frac{\tilde{f}_{W W}}{\Lambda^{2}} \Phi^{\dagger} \hat{\tilde{W}}^{\mu \nu} \hat{W}_{\mu \nu} \Phi ; \\
\tilde{O}_{B W} & =\frac{\tilde{f}_{B W}}{\Lambda^{2}} \Phi^{\dagger} \hat{\tilde{B}}^{\mu \nu} \hat{W}_{\mu \nu} \Phi,
\end{aligned}
$$

Here $\Lambda$ is the cut-off scale above which the high scale physics sets in. For the purpose of this study $\Lambda$ is taken to be $1 \mathrm{TeV}$. Since for the LHC observables, $\tilde{f} i / \Lambda^{2}(i=B, W, W W$ etc. $)$ is the effective parameter that gets constrained, the global fit constraints can be predicted for any value of $\Lambda$, with the constraints on the parameters $\tilde{f} i$ 's being appropriately scaled. However, for the constraints coming from EDM measurements, one finds that EDMs have an explicit cut-off dependence other than the one coming via the parameters $\tilde{f} i / \Lambda^{2}$. This dependence coming from loop contributions is logarithmic in nature 65].

However, all five of these CP-violating operators are not independent, and are related by the following constraint equations, which arise from using the SM equations of motion: 1

$$
\begin{aligned}
2 \tilde{O}_{B} & =\frac{\tilde{f}_{B}}{\tilde{f}_{B B}} \tilde{O}_{B B}+\frac{\tilde{f}_{B}}{\tilde{f}_{B W}} \tilde{O}_{B W}, \\
2 \tilde{O}_{W} & =\frac{\tilde{f}_{W}}{\tilde{f}_{W W}} \tilde{O}_{W W}+\frac{\tilde{f}_{W}}{\tilde{f}_{B W}} \tilde{O}_{B W}
\end{aligned}
$$

\footnotetext{
${ }^{1}$ We thank Wouter Dekens and Jordy de Vries for helpful comments on this matter.
} 
The above set of constraints in Eq. 2 entails only three of the five operators in Eq. 1 to be independent of each other. For the purpose of our study we take $\tilde{O}_{B}, \tilde{O}_{W}$ and $\tilde{O}_{W W}$ to be comprising an independent set of CP-violating gauge-Higgs operators. These operators give rise to the CP-odd $V V h$ interaction vertices of following form,

$$
\tilde{\mathcal{L}}_{V V h}=\frac{g m_{W}}{\Lambda^{2}} \tilde{C}_{V V h}\left[\epsilon_{\mu \nu \alpha \beta} k_{1}^{\alpha} k_{2}^{\beta}\right] V^{\mu}\left(k_{1}\right) V^{\nu}\left(k_{2}\right) h(k),
$$

where $V=W, Z, \gamma$. The coefficients $\tilde{C}_{V V h}$ denote the effective coupling strengths of CP-odd $V V h$ couplings and, are listed in Table 1. The $V V h$ interaction vertices arising from corresponding CP-even operators are derived in Ref. 62].

Our aim in the present work is to include scenarios where both CP-odd and-even operators are present, and to discriminate between them via appropriately defined asymmetry parameters. We demonstrate this by turning on the CP-even operator,

$$
O_{W W}=\frac{f_{W W}}{\Lambda^{2}} \Phi^{\dagger} \hat{W}^{\mu \nu} \hat{W}_{\mu \nu} \Phi
$$

which contributes to all the $V V h$ vertices. The additional interaction terms thus playing a role in our analysis have the following form 62]

$$
\mathcal{L}_{V V h}=\frac{g m_{W}}{\Lambda^{2}} C_{V V h}\left[k_{1}{ }^{\nu} k_{2}{ }^{\mu}-g^{\mu \nu}\left(k_{1} . k_{2}\right)\right] V_{\mu}\left(k_{1}\right) V_{\nu}\left(k_{2}\right) h(k) .
$$

The coupling strengths of CP-even $V V h$ couplings are given by

$$
C_{W W h}=-2 f_{W W} ; C_{Z Z h}=-2 c_{W}^{2} f_{W W} ; C_{\gamma \gamma h}=-2 s_{W}^{2} f_{W W} ; C_{\gamma Z h}=-2 c_{W} s_{W} f_{W W}
$$

which are similar to the corresponding CP-odd couplings receiving contributions from $\tilde{O}_{W W}$ operator.

In 65] we derived limits on the parameters of the CP-odd operators using the collider data (from LEP and LHC) and measurements of electron and neutron EDMs. These limits were obtained taking mainly two parameters $(2 \mathrm{P})$ and three parameters $(3 \mathrm{P})$ nonzero at a time. The limits on parameters were further used to constrain the effective CP-odd $V V h$ couplings which are listed in Table 2.

As has been already stated, these constraints can get relaxed when we keep a linearly independent set of CP-violating gauge-Higgs operators to be non-zero at the same time along with the other fermion-Higgs dimension-6 operators (which contribute to fermionic EDMs), and also include at least some of their CP-conserving counterparts. Such operators can be obtained directly by extending those listed in [58, Table 2. While the CP-violating ones contribute to EDMs, they do not affect the phenomenology of $V V h$ interactions. The consequent enlargement of the parameter space, however, relaxes the otherwise stringent constraints from EDMs.

The allowed values of the coefficients of the dimension- 6 operators apparently threaten perturbative unitarity in $V_{L} V_{L} \rightarrow V_{L} V_{L}(V=W, Z)$ at scales of the order of few TeV. The exact value of this scale depends on the specific choice of the operators involved and the corresponding anomalous couplings they generate [66,67]. However, the effective operators are not reliable at such scales. There one has to include the actual heavy fields of mass $\mathcal{O}(\Lambda)$ (for weakly coupled ultraviolet completion), which are integrated out to obtain the effective coupling terms. These additional degrees of freedom are trusted to restore unitarity when the full theory is switched on. In the context of the study presented here, the range of $\tilde{C}_{Z Z h}$ is varied upto $\mathcal{O}(70)$. Even though 


\begin{tabular}{|c|c|}
\hline Coupling & Effective coupling strength \\
\hline$\tilde{C}_{W W h}$ & $\left(-\tilde{f}_{W}-2 \tilde{f}_{W W}\right)$ \\
\hline$\tilde{C}_{Z Z h}$ & $-1 / c_{W}^{2}\left(c_{W}^{2} \tilde{f}_{W}+s_{W}^{2} \tilde{f}_{B}+2 c_{W}^{4} \tilde{f}_{W W}\right)$ \\
\hline$\tilde{C}_{\gamma \gamma h}$ & $-2 s_{W}^{2} \tilde{f}_{W W}$ \\
\hline$\tilde{C}_{\gamma Z h}$ & $t_{W} / 2\left(-\tilde{f}_{W}+\tilde{f}_{B}-4 c_{W}^{2} \tilde{f}_{W W}\right)$ \\
\hline
\end{tabular}

Table 1: CP-odd $V V h$ coupling factors and their effective strengths in terms of operator coefficients $\tilde{f}_{B}, \tilde{f}_{W}$ and $\tilde{f}_{W W}$.

$\tilde{C}_{Z Z h}$ is varied upto this range, the effective coupling parameter that will decide the perturbativity of the loop expanson is given by [36],

$$
\tilde{b}=\frac{g m_{W} v}{2 \Lambda^{2}} \tilde{C}_{Z Z h}
$$

Here, $v=246 \mathrm{GeV}$ is the Higgs vacuum expectation value. Thus even for $\tilde{C}_{Z Z h} \lesssim \mathcal{O}(70), \tilde{b} \lesssim \mathcal{O}(1)$ and the loop expansion in terms of $\tilde{b}$ remains perturbative.

\section{Isolating the effect of CP-odd couplings}

In any elementary particle scattering process, the size and shape of certain kinematic distributions involving leptons and jets can change non-trivially with respect to the purely standard model prediction in the presence of both CP-odd and-even higher dimensional operators. Some of these have been studied mostly in purely phenomenological formulations, i.e., without working in terms of gauge invariant dimension-6 operators or without performing a realistic simulation of events ( $[8,11,12,19,28,31,32,34,38,39]$ ). For generic observables like $p_{T}$ and rapidity distributions which are affected by both CP-even as well as CP-odd couplings, it may not be possible to separate the effect of the two couplings with sufficient statistical significance. In other words, for values of anomalous couplings, consistent with the available experimental data, it would require analysing a huge amount of data/events to confirm the effect of CP-odd couplings on top of the effect of CPeven couplings using generic observables. Therefore, it is more economical to work with observables which are unambiguously sensitive to the definite CP-propertry of the couplings.

In the present work, we are interested in CP-odd observables. The CP-odd observables can be categorised as $\hat{T}$-even or $\hat{T}$-odd type 2 . Unlike the $\hat{T}$-even observables which require a nonzero absorptive/unitary phase to quantify the CP-violation, the $\hat{T}$-odd observables do not require

\footnotetext{
${ }^{2} \hat{T}$ is the naive time reversal transformation that flips the sign of the particle spin and momenta, without interchanging the initial and final states.
} 


\begin{tabular}{|c|c|c|c|c|}
\hline Couplings & \multicolumn{2}{|c|}{ LHC data } & \multicolumn{2}{c|}{ EDM } \\
\cline { 2 - 5 } & 2P case & 3P case & 2P case & 3P case \\
\hline$\left|\tilde{C}_{W W h}\right|$ & $0-60$ & $0-60$ & $0-0.17$ & $0-55$ \\
\hline$\left|\tilde{C}_{Z Z h}\right|$ & $25-80$ & $25-80$ & $0.11-0.20$ & $0.15-33$ \\
\hline$\left|\tilde{C}_{\gamma \gamma h}\right|$ & $0-0.8$ & $0-0.8$ & $0-0.16$ & $0.02-52$ \\
\hline$\left|\tilde{C}_{\gamma z h}\right|$ & $15-25$ & $15-25$ & $0.03-0.25$ & $0.05-110$ \\
\hline
\end{tabular}

Table 2: Limits on CP-odd coupling strengths from LHC data and EDM measurements for $\Lambda=1$ $\mathrm{TeV} .2 \mathrm{P}$ and $3 \mathrm{P}$ stand for two parameter nonzero and three parameter nonzero cases respectively.

any absorptive phase and can be calculated at tree-level. The triple cross products constructed using the particle momenta/spin in a given process can serve as $\hat{T}$-odd observables; thus they need not be driven by any absorptive phase. A non-zero expectation value of such a CP-odd and $\hat{T}$-odd observable would imply CP-violation at the Lagrangian level [68. In practice, we work with asymmetries, constructed out of such observables, which are unlikely to be faked by the SM or other CP-even couplings via statistical fluctuation. In this section, we quantitatively establish such characteristics of the chosen observables after a realistic simulation of a suitable process at the LHC.

\subsection{Signal and backgrounds}

With the above considerations in view, we take up the analysis of the process, $p p \rightarrow Z h \rightarrow \ell^{+} \ell^{-} b \bar{b}$ with $\ell=e, \mu$. We consider the decay of Higgs to $b \bar{b}$ final state to ensure sufficient event rates. The final state is required to have two opposite sign leptons and exactly two b-tagged jets. The protonproton collisions are simulated at the center-of-mass energy of $14 \mathrm{TeV}$. In presence of dimension- 6 operators, this process also receives contribution from a tree-level diagram involving $\gamma Z h$ vertex, which is not present in the SM. Thus there is an interference of the two $s$-channel diagrams involving the $Z Z h$ and $\gamma Z h$ vertices at the amplitude level. This is because the same gauge-Higgs operators that give rise to the anomalous $C_{Z Z h}$ coupling also give rise to the $C_{\gamma Z h}$ interaction [Table 1].

Throughout our analysis we consider $M_{h}=126 \mathrm{GeV}$. We implement the anomalous couplings in Madgraph [69] using the package FeynRules [70]. The cross sections are calculated using nn23lo1 [71] parton distribution functions with default settings for renormalization and factorization scales. While the parton level events are generated in Madgraph, the Pythia 72 switch is turned on to incorporate showering, hadronisation and initial and final state radiation (ISR/FSR) effects. The HEP file obtained from this interfacing is passed to Delphes-3.1.2 [73] which gives a ROOT [74] file as output. Jets are formed using the anti-kt algorithm in Delphes with a cone size of 0.6. A $p_{T}$ dependent tagging efficiency is employed for $b$-jets, namely, $0.7-0.5$ for $p_{T}$ in the range $20-500 \mathrm{GeV}$. We also use a mis-tagging efficiency of 0.001 for light jets and a $p_{T}$ dependent 
mis-tagging efficiency for $c$-induced jets, which varies from $0.14-0.08$ for $20<p_{T}<500 \mathrm{GeV}$. The ROOT file thus obtained is fed to the analysis code to generate the distributions.

We start with following basic cuts on the transverse momentum, rapidity and separation of leptons $(\ell)$ and jets $(J)$, where $J=j, b ; j$ being the light quark jets and $b$ corresponds to the $b$-induced jets.

1. $p_{T_{J, \ell}}>20 \mathrm{GeV}$ and $\left|\eta_{J, \ell}\right|<2.5$ : We demand that our final state leptons and jets should lie in the central rapidity region each with a minimum transverse momentum of $20 \mathrm{GeV}$.

2. $\Delta R_{J J, J \ell}>0.4$ : We demand that both the jets should be well separated from each other as well as from leptons.

3. $\Delta R_{\ell \ell}>0.2$ : There will be sufficiently large opening angle between the two leptons.

The SM cross section for the signal after the generation level cuts is $12.4 \mathrm{fb}$.

The major background to the signal comes from the following SM processes:

1. $p p \rightarrow b \bar{b} \ell^{+} \ell^{-}(\mathrm{QCD})$

2. $p p \rightarrow t \bar{t} \rightarrow b \bar{b} \ell^{+} \ell^{-} \nu \bar{\nu}$

3. $p p \rightarrow j j \ell^{+} \ell^{-}(\mathrm{QCD})$

4. $p p \rightarrow Z \ell^{+} \ell^{-} \rightarrow b \bar{b} \ell^{+} \ell^{-}$(EW)

To suppress the above sources of the background, we employ the following additional selection cuts :

1. $p_{T_{J}}>50 \mathrm{GeV}$

2. $105<M_{J J}<130 \mathrm{GeV}$

3. $\left|M_{l l}-M_{Z}\right|<15 \mathrm{GeV}$

4. The $p_{T}$ of the reconstructed $Z$ boson is taken to be $>150 \mathrm{GeV}$

The asymmetrical cut on the invariant mass of the two $b$-tagged jets $\left(M_{J J}\right)$ in the final state around the peak ( approximately $116 \mathrm{GeV}$ ) is helpful in suppressing backgrounds from $p p \rightarrow b \bar{b} \ell^{+} \ell^{-}$and $p p \rightarrow Z \ell^{+} \ell^{-} \rightarrow b \bar{b} \ell^{+} \ell^{-}$processes. We have taken such a window for the invariant mass of the two $b$-tagged jets because owing to the effects of hadronisation and showering, the peak of the invariant mass distribution shifts from $M_{h}$ to about $116 \mathrm{GeV}$. Thus we gain in terms of signal events by choosing such an invariant mass cut. Further, requiring exactly two $b$-tagged jets in the final state significantly reduces the background coming from $p p \rightarrow j j \ell^{+} \ell^{-}$.

In order to suppress the $t \bar{t}$ backgrounds, use has been made of the fact that our signal events are hermetic in the ideal situation. In contrast, similar final states from the $t \bar{t}$ background will be necessarily associated with missing- $E_{T}$. Thus we propose to distinguish the signal by demanding $E_{T}<20 \mathrm{GeV}$ [36]. Moreover, no missing- $p_{T}$ pointing centrally is ensured by choosing $|\not| x \mid>2.5$, where $\not$ stands for the polar direction of the transverse momentum imbalance. 


\subsection{Observables}

Next, we discuss two CP-odd, $\hat{T}$-odd quantities used in the rest of the analysis to isolate the effect of CP-odd couplings from the CP-even ones. These observables are constructed using the 3-momentum information of the final state particles. The first one, also discussed in Refs. 35, 36] is given by,

$$
\mathcal{O}_{1}=\frac{\left(\vec{p}_{+} \times \vec{p}_{-}\right) \cdot \hat{z}}{\left|\vec{p}_{+} \times \vec{p}_{-}\right|} \operatorname{sign}\left[\left(\vec{p}_{+}-\vec{p}_{-}\right) \cdot \hat{z}\right]
$$

Here $\vec{p}_{+}$and $\vec{p}_{-}$are the 3 -momenta of $\ell^{+}$and $\ell^{-}$respectively, and $\hat{z}$ is the unit vector along the incoming quark(or anti-quark) direction or the collision axis. The factor $\operatorname{sign}\left[\left(\vec{p}_{+}-\vec{p}_{-}\right) . \hat{z}\right]$ is the sign of the difference of the momentum projections of the outgoing leptons along the $\hat{z}$ axis. Since the dot product with the $\hat{z}$ unit vector occurs twice in the definition of the observable, the observable is rendered independent of the choice of the direction of the incoming quark momentum. This is important in the context of LHC, as there is ambiguity associated with the momentum direction of the initial quark with respect to which proton it is from. The definition of $\mathcal{O}_{1}$ removes this ambiguity and makes it uniquely measurable.

The second observable that we consider uses $\vec{p}_{j_{1}}$ and $\vec{p}_{j_{2}}$, the three-momenta of the two $b$-tagged jets in addition to the oppositely signed leptons in the final state and is given as:

$$
\mathcal{O}_{2}=\frac{\left(\left(\vec{p}_{j_{1}}+\vec{p}_{j_{2}}\right) \times\left(\vec{p}_{+}-\vec{p}_{-}\right)\right) \cdot \hat{z}}{\left|\left(\vec{p}_{j_{1}}+\vec{p}_{j_{2}}\right) \times\left(\vec{p}_{+}-\vec{p}_{-}\right)\right|} \operatorname{sign}\left[\left(\vec{p}_{+}-\vec{p}_{-}\right) \cdot \hat{z}\right],
$$

These observables are CP-odd and $\hat{T}$-odd, and thus generated from the dispersive part of the amplitude. It should also be noted that, since the in-state partons from the two colliding proton beams do not always carry equal momenta, the lab frame and the center of mass $(\mathrm{cm})$ frame for the colliding partons are in general different. Thus, except for the $\mathrm{cm}$ frame i.e. when the two initial partons come with the same $x$-value, where $\vec{p}_{j_{1}}+\vec{p}_{j_{2}}+\vec{p}_{+}+\vec{p}_{-}=0$, the observables $\mathcal{O}_{1}$ and $\mathcal{O}_{2}$ are distinct. Also, due to the showering effects, an exact momentum balance between the final state $b$-jets and leptons does not hold true. The difference in the observables is evident from their distributions. By definition, each of them ranges from -1.0 to +1.0 .

The distinguishability of the CP-odd operators is quantified more effectively in terms of the asymmetries $A_{i}$ defined as:

$$
A_{i}=\frac{\sigma\left(\mathcal{O}_{i}>0\right)-\sigma\left(\mathcal{O}_{i}<0\right)}{\sigma\left(\mathcal{O}_{i}>0\right)+\sigma\left(\mathcal{O}_{i}<0\right)}
$$

We show in Figs. 1 1and 2 histograms of the differential cross section distributions corresponding to observables $\mathcal{O}_{1}$ and $\mathcal{O}_{2}$ respectively. In both the figures, to tune in the CP-even Lagrangian, we have taken the benchmark value $f_{W W}=-3.0$ [75] which corresponds to $C_{Z Z h}=4.62$ and $C_{\gamma Z h}=2.53$. As for the CP-odd terms, we take the benchmark point as $\tilde{f}_{B}=\tilde{f}_{W}=-30.77$ such that $\tilde{C}_{Z Z h}=40.0$ and $\tilde{C}_{\gamma Z h}=0.0$ [Table 1] ]. Both of these benchmarks are consistent with existing constraints [65, 75]. The histograms in the two figures are indicative of the induced asymmetry in the distributions for both $\mathcal{O}_{1}$ and $\mathcal{O}_{2}$ when the CP-violating coupling is turned on. It is important to note that unlike total rates and other CP-even observables which depend on CP-odd couplings quadratically, a comparison of distributions from second and third panel confirms that these observables have linear dependence on $\tilde{C}_{Z Z h}$ coupling in the numerator of the expression for the asymmetry [Eq. 10]. The qualitative features of the histograms remain similar when the $\tilde{C}_{\gamma Z h}$ coupling is also taken to be non-zero and interference effects of both $\tilde{C}_{Z Z h}$ and $\tilde{C}_{\gamma Z h}$ couplings are considered. 


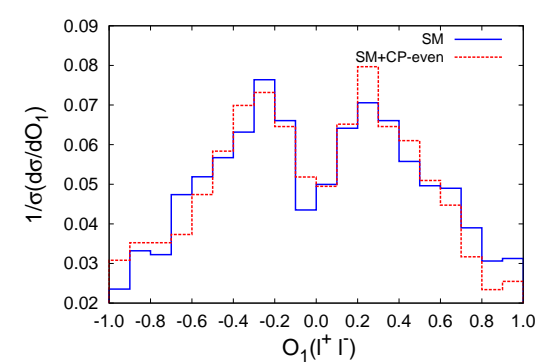

(a)

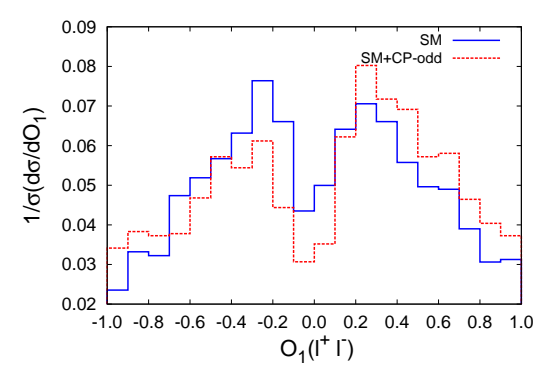

(b)

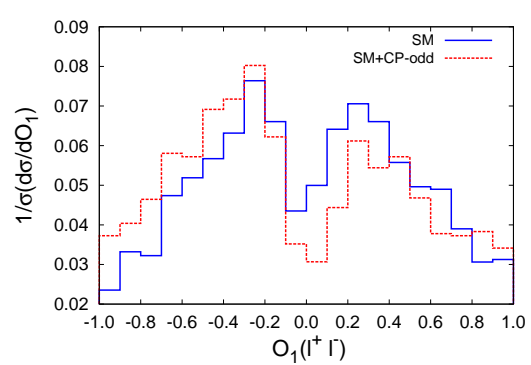

(c)

Figure 1: Differential cross-section distributions for $\mathcal{O}_{1}$ at $\sqrt{s}=14 \mathrm{TeV}$. (a) SM vs SM $+\mathrm{CP}$-even case with $C_{Z Z h}=4.62$ and $C_{\gamma Z h}=2.53$. (b) SM vs SM + CP-odd case with $\tilde{C}_{Z Z h}=40.0$ and $\tilde{C}_{\gamma Z h}=0.0$. (c) We reverse the sign of the CP-odd coupling with $\tilde{C}_{Z Z h}=-40.0$ and $\tilde{C}_{\gamma Z h}=0.0$.

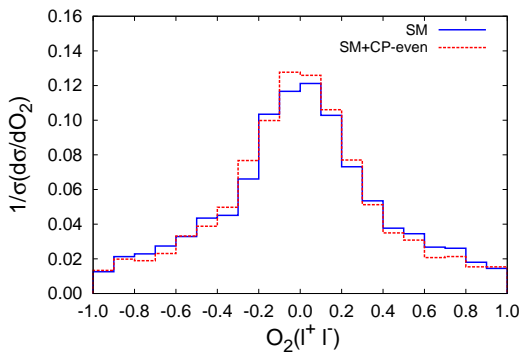

(a)

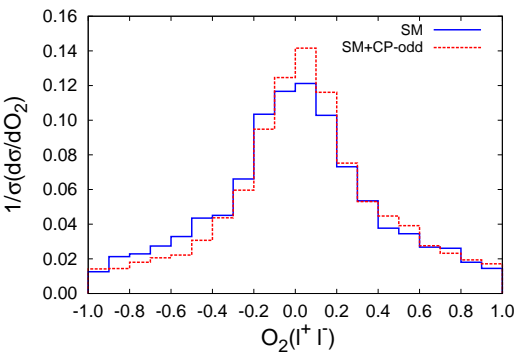

(b)

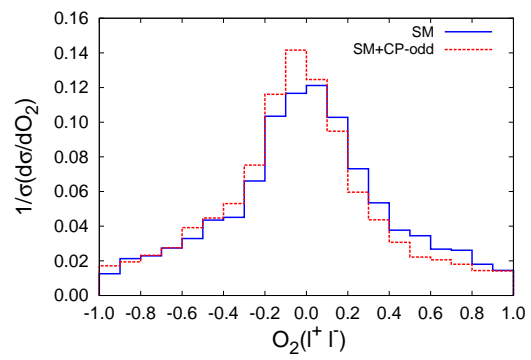

(c)

Figure 2: Differential cross-section distributions for $\mathcal{O}_{2}$ at $\sqrt{s}=14 \mathrm{TeV}$. (a) SM vs SM + CP-even case with $C_{Z Z h}=4.62$ and $C_{\gamma Z h}=2.53$. (b) $\mathrm{SM}$ vs $\mathrm{SM}+\mathrm{CP}$-odd case with $\tilde{C}_{Z Z h}=40.0$ and $\tilde{C}_{\gamma Z h}=0.0$. (c) We reverse the sign of the CP-odd coupling with $\tilde{C}_{Z Z h}=-40.0$ and $\tilde{C}_{\gamma Z h}=0.0$.

\subsection{Asymmetries vs couplings}

To assess the possibility of seeing the signatures of CP-violating physics in isolation, we need to calculate the statistical significance at which one can establish the existence of non-vanishing asymmetry over and above the CP-conserving effects coming from the SM background. If $N_{S}$ is the number of signal events and $N_{B}$ the number of background events, then the observed asymmetry $A_{i}^{\text {obs }}$, corresponding to observable $\mathcal{O}_{i}$ at the detector is related to the theoretical asymmetry in the signal distribution i.e. $A_{i}$, given in Eq. (10) by the following relation:

$$
A_{i}^{\text {obs }}=A_{i}\left(\frac{N_{S}}{N_{S}+N_{B}}\right)
$$

The statistical error in the observed asymmetry is given by,

$$
\Delta A_{i}^{\text {obs }}=\frac{1}{\sqrt{N_{S}+N_{B}}}=\frac{1}{\sqrt{N}},
$$

where $N=N_{S}+N_{B}$ is the total number of signal and background events. Using the above information, the significance $S$, associated with an observed asymmetry is given by [39],

$$
S=A_{i}^{\mathrm{obs}} \sqrt{N}=A_{i} \frac{N_{S}}{\sqrt{N}}
$$




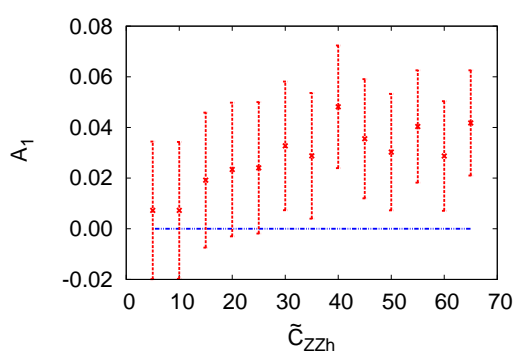

(a) $\mathcal{L}=1 \mathrm{ab}^{-1}$

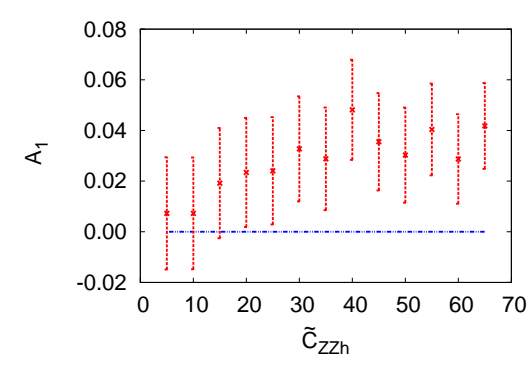

(b) $\mathcal{L}=1.5 \mathrm{ab}^{-1}$

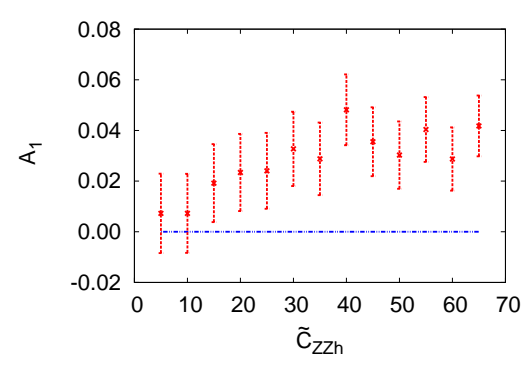

(c) $\mathcal{L}=3 \mathrm{ab}^{-1}$

Figure 3: The asymmetry vs. coupling plot for the variable $\mathcal{O}_{1}$ at Luminosities $1 \mathrm{ab}^{-1}, 1.5 \mathrm{ab}^{-1}$ and $3 \mathrm{ab}^{-1}$, plotted for varying $\tilde{C}_{Z Z h}$. The benchmark points are chosen such that $\tilde{C}_{\gamma Z h}=0.0$. Statistical uncertainties are shown as error bars for different benchmark points. The zero line is shown to illustrate the offset of the non-vanishing asymmetry against the SM and CP-even case, for which a CP-odd observable has no asymmetry.

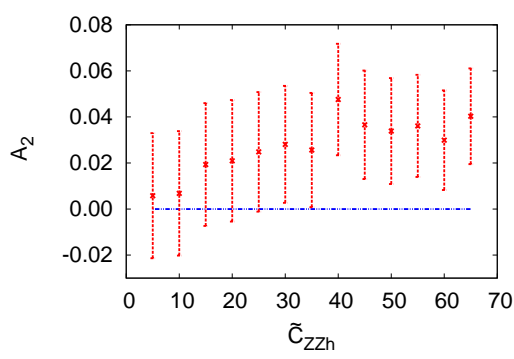

(a) $\mathcal{L}=1 \mathrm{ab}^{-1}$

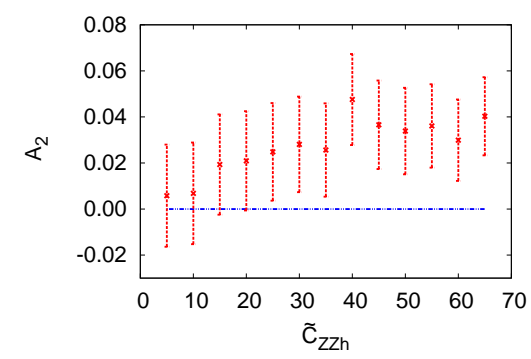

(b) $\mathcal{L}=1.5 \mathrm{ab}^{-1}$

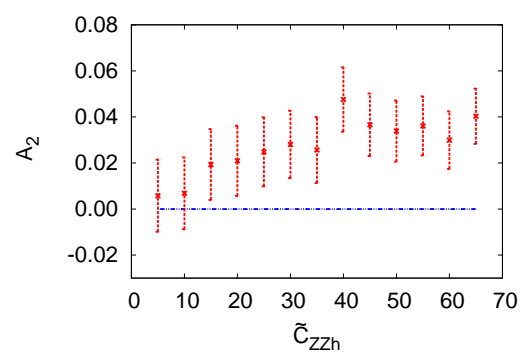

(c) $\mathcal{L}=3 \mathrm{ab}^{-1}$

Figure 4: The asymmetry vs. coupling plot for the variable $\mathcal{O}_{2}$ at Luminosities $1 \mathrm{ab}^{-1}, 1.5 \mathrm{ab}^{-1}$ and $3 \mathrm{ab}^{-1}$, plotted for varying $\tilde{C}_{Z Z h}$. The benchmark points are chosen such that $\tilde{C}_{\gamma Z h}=0.0$.

With this in mind, we plot the variation of the asymmetry against the value of the coupling parameter $\tilde{C}_{Z Z h}$ as shown in Figures 3 to 6 , Figures 3 and 4 show the variation of asymmetry for observables $\mathcal{O}_{1}$ and $\mathcal{O}_{2}$ respectively. For both of them we vary $\tilde{C}_{Z Z h}$ keeping $\tilde{C}_{\gamma Z h}=0.0$, wheres Figures $[5$ and 6 show the asymmetry plots for the same observables, although this time we fix the $\tilde{C}_{\gamma Z h}$ coupling at 1.5. For each of the Figures 3 to 6 we present the error bars on the asymmetries for the various benchmark points. We have not included any specific values of systematic uncertainties which can in principle change the asymmetries by a few percent.

Our calculations yield near vanishing asymmetry for SM and CP-even cases, which are consistent with zero within $0.5 \sigma$ for the chosen integrated luminosities. Looking at Figures 3 to 6 one can infer that the statistical significance improves as we go from a luminosity reach of $1 \mathrm{ab}^{-1}$ to $3 \mathrm{ab}^{-1}$. For example, in Fig. 3, we see that for $\tilde{C}_{Z Z h}=40.0$, the sensitivity improves from $2 \sigma$ to about $4 \sigma$ in going from $1 \mathrm{ab}^{-1}$ to $3 \mathrm{ab}^{-1}$. Another important feature to notice is that the asymmetry does not monotonically increase as we increase the magnitude of the coupling parameter. This is due to the fact that for higher values of couplings, the higher order terms, i.e. beyond the interference term linear in $\tilde{C}_{Z Z h}$, become influential. This is corroborated by the marginal decrease in the asymmetry curve beyond $\tilde{C}_{Z Z h}=40.0$ in Figures 3 and 4 . The analysis done here agrees at the parton level to the work presented in [36], but once we fold in the ISR/FSR and detector effects, the statistical significance gets lowered in comparison to what one gets for the parton level analysis. 


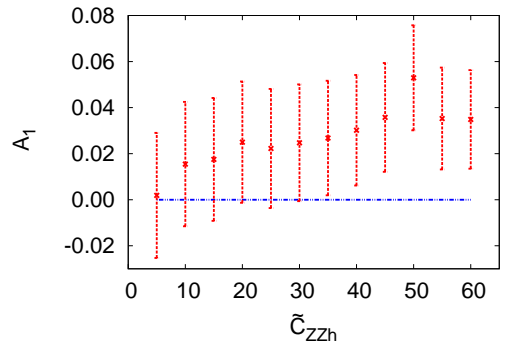

(a) $\mathcal{L}=1 \mathrm{ab}^{-1}$

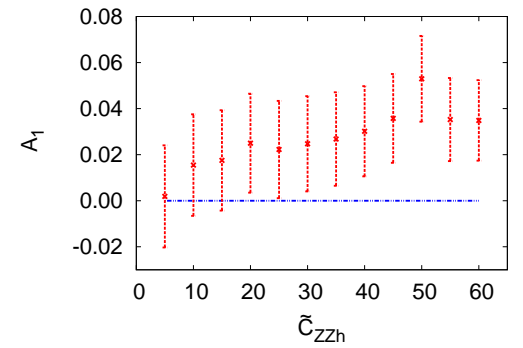

(b) $\mathcal{L}=1.5 \mathrm{ab}^{-1}$

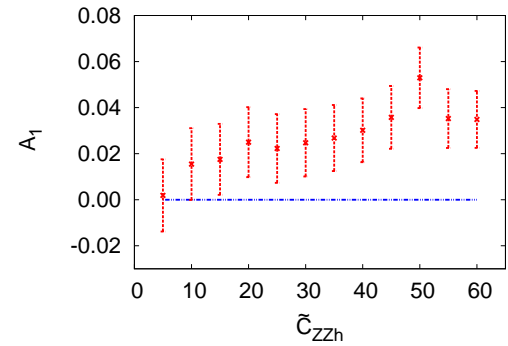

(c) $\mathcal{L}=3 \mathrm{ab}^{-1}$

Figure 5: The asymmetry vs. coupling plot for the variable $\mathcal{O}_{1}$ at Luminosities $1 \mathrm{ab}^{-1}, 1.5 \mathrm{ab}^{-1}$ and $3 \mathrm{ab}^{-1}$, plotted for varying $\tilde{C}_{Z Z h}$. The benchmark points are chosen such that $\tilde{C}_{\gamma Z h}=1.5$.

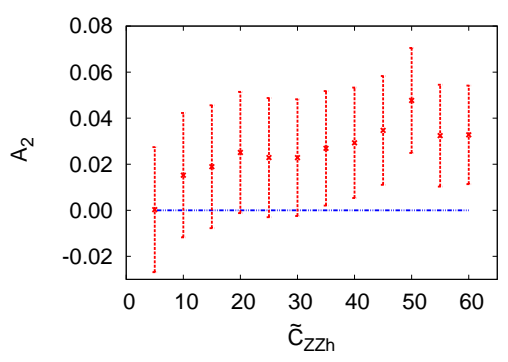

(a) $\mathcal{L}=1 \mathrm{ab}^{-1}$

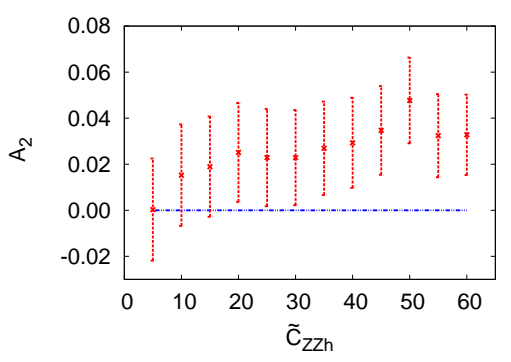

(b) $\mathcal{L}=1.5 \mathrm{ab}^{-1}$

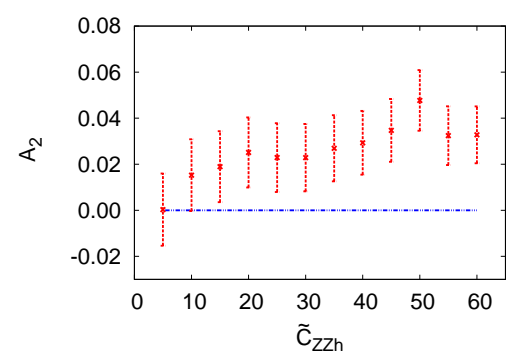

(c) $\mathcal{L}=3 \mathrm{ab}^{-1}$

Figure 6: The asymmetry vs. coupling plot for the variable $\mathcal{O}_{2}$ at Luminosities $1 \mathrm{ab}^{-1}, 1.5 \mathrm{ab}^{-1}$ and $3 \mathrm{ab}^{-1}$, plotted for varying $\tilde{C}_{Z Z h}$. The benchmark points are chosen such that $\tilde{C}_{\gamma Z h}=1.5$. 


\section{Summary and Conclusions}

We have looked for experimentally realizing the effects of CP-violating operators that arise from gauge-invariant dimension- $6 V V h$ couplings. Values of the couplings consistent with electric dipole moments as well as global fits of the LHC data are taken. We have studied higgstrahlung process $(p p \rightarrow Z h)$ in presence of both CP-even and CP-odd couplings. We have included effects of parton showers and also factored in detector simulation, to make our estimates as realistic as possible. The presence of non vanishing asymmetries over and above the statistical errors is seen as the litmus test for testing the presence of $\mathrm{CP}$-violating new physics. To this end, we have shown that for SM and CP-conserving dimension-6 operators, observables which are both $\mathrm{CP}$ and $\hat{T}$ odd do not yield any asymmetry in their kinematic distributions. But the presence of CP-violating gaugeHiggs operators is seen to give rise to non-vanishing asymmetries for such observables, thus clearly demarcating the presence of CP-violating high scale physics. We have been able to show that for a benchmark value of $\tilde{C}_{Z Z h}=40.0$, asymmetry can be established at about $4 \sigma$ level with $3 \mathrm{ab}^{-1}$ in the $14 \mathrm{TeV}$ run of the LHC.

\section{Acknowledgements}

We thank Shankha Banerjee, Jyotiranjan Beuria, Tanumoy Mandal, Subhadeep Mondal, Pratishruti Saha and Juhi Dutta for fruitful discussions. The work of SD, BM and AS is partially supported by funding available from the Department of Atomic Energy, Government of India, for the Regional Centre for Accelerator-based Particle Physics (RECAPP), Harish-Chandra Research Institute. SD acknowledges the hospitality of Indian Association for the Cultivation of Science, Kolkata. 


\section{References}

[1] G. Aad et al. [ATLAS Collaboration], Phys. Lett. B 716, 1 (2012) [arXiv:1207.7214 [hep-ex]].

[2] S. Chatrchyan et al. [CMS Collaboration], Phys. Lett. B 716, 30 (2012) arXiv:1207.7235 [hep-ex]].

[3] Y. Gao, A. V. Gritsan, Z. Guo, K. Melnikov, M. Schulze and N. V. Tran, Phys. Rev. D 81, 075022 (2010) doi:10.1103/PhysRevD.81.075022 [arXiv:1001.3396 [hep-ph]].

[4] S. Y. Choi, D. J. Miller, M. M. Muhlleitner and P. M. Zerwas, Phys. Lett. B 553, 61 (2003) doi:10.1016/S0370-2693(02)03191-X hep-ph/0210077].

[5] A. De Rujula, J. Lykken, M. Pierini, C. Rogan and M. Spiropulu, Phys. Rev. D 82, 013003 (2010) doi:10.1103/PhysRevD.82.013003 [arXiv:1001.5300 [hep-ph]].

[6] R. Boughezal, T. J. LeCompte and F. Petriello, arXiv:1208.4311 [hep-ph].

[7] D. Stolarski and R. Vega-Morales, Phys. Rev. D 86, 117504 (2012) doi:10.1103/PhysRevD.86.117504 [arXiv:1208.4840 [hep-ph]].

[8] A. Djouadi, R. M. Godbole, B. Mellado and K. Mohan, Phys. Lett. B 723, 307 (2013) doi:10.1016/j.physletb.2013.04.060 [arXiv:1301.4965 [hep-ph]].

[9] R. Godbole, D. J. Miller, K. Mohan and C. D. White, Phys. Lett. B 730, 275 (2014) doi:10.1016/j.physletb.2014.01.069 [arXiv:1306.2573 [hep-ph]].

[10] J. Ellis, V. Sanz and T. You, Eur. Phys. J. C 73, 2507 (2013) doi:10.1140/epjc/s10052-0132507-6 [arXiv:1303.0208 [hep-ph]].

[11] Y. Sun, X. F. Wang and D. N. Gao, Int. J. Mod. Phys. A 29, 1450086 (2014) doi:10.1142/S0217751X14500869 [arXiv:1309.4171 [hep-ph]].

[12] I. Anderson et al., Phys. Rev. D 89, no. 3, 035007 (2014) doi:10.1103/PhysRevD.89.035007 arXiv:1309.4819 [hep-ph]].

[13] C. Delaunay, G. Perez, H. de Sandes and W. Skiba, Phys. Rev. D 89, no. 3, 035004 (2014) doi:10.1103/PhysRevD.89.035004 [arXiv:1308.4930 [hep-ph]].

[14] F. Maltoni, K. Mawatari and M. Zaro, Eur. Phys. J. C 74, no. 1, 2710 (2014) doi:10.1140/epjc/s10052-013-2710-5 [arXiv:1311.1829 [hep-ph]].

[15] M. B. Gavela, J. Gonzalez-Fraile, M. C. Gonzalez-Garcia, L. Merlo, S. Rigolin and J. Yepes, JHEP 1410, 44 (2014) doi:10.1007/JHEP10(2014)044 [arXiv:1406.6367 [hep-ph]].

[16] S. Banerjee, S. Mukhopadhyay and B. Mukhopadhyaya, Phys. Rev. D 89, no. 5, 053010 (2014) doi:10.1103/PhysRevD.89.053010 [arXiv:1308.4860 [hep-ph]].

[17] G. Amar, S. Banerjee, S. von Buddenbrock, A. S. Cornell, T. Mandal, B. Mellado and B. Mukhopadhyaya, JHEP 1502, 128 (2015) doi:10.1007/JHEP02(2015)128 arXiv:1405.3957 [hep-ph]].

[18] J. Ellis, V. Sanz and T. You, JHEP 1407, 036 (2014) doi:10.1007/JHEP07(2014)036 arXiv:1404.3667 [hep-ph]]. 
[19] N. Desai, D. K. Ghosh and B. Mukhopadhyaya, Phys. Rev. D 83, 113004 (2011) [arXiv:1104.3327 [hep-ph]].

[20] S. Bolognesi, Y. Gao, A. V. Gritsan, K. Melnikov, M. Schulze, N. V. Tran and A. Whitbeck, Phys. Rev. D 86, 095031 (2012) doi:10.1103/PhysRevD.86.095031 [arXiv:1208.4018 [hep-ph]].

[21] Y. Chen, A. Falkowski, I. Low and R. Vega-Morales, Phys. Rev. D 90, no. 11, 113006 (2014) doi:10.1103/PhysRevD.90.113006 [arXiv:1405.6723 [hep-ph]].

[22] CMS Collaboration Collaboration, Constraints on anomalous $H V V$ interactions using $H$ to 4 l decays, Tech. Rep. CMS-PAS-HIG-14-014, CERN, Geneva, 2014.

[23] V. Khachatryan et al. [CMS Collaboration], Phys. Rev. D 92, no. 1, 012004 (2015) doi:10.1103/PhysRevD.92.012004 [arXiv:1411.3441 [hep-ex]].

[24] CMS Collaboration Collaboration, Constraints on Anomalous $H W W$ Interactions using Higgs boson decays to $W^{+} W^{-}$in the fully leptonic final state, Tech. Rep. CMS-PAS-HIG-14012, CERN, Geneva, 2014.

[25] T. Plehn, D. L. Rainwater and D. Zeppenfeld, Phys. Rev. Lett. 88, 051801 (2002) doi:10.1103/PhysRevLett.88.051801 [hep-ph/0105325].

[26] V. Hankele, G. Klamke, D. Zeppenfeld and T. Figy, Phys. Rev. D 74, 095001 (2006) doi:10.1103/PhysRevD.74.095001 hep-ph/0609075].

[27] D. J. Miller, S. Y. Choi, B. Eberle, M. M. Muhlleitner and P. M. Zerwas, Phys. Lett. B 505, 149 (2001) doi:10.1016/S0370-2693(01)00317-3 hep-ph/0102023.

[28] T. Han and J. Jiang, Phys. Rev. D 63, 096007 (2001) hep-ph/0011271].

[29] S. S. Biswal, D. Choudhury, R. M. Godbole and Mamta, Phys. Rev. D 79, 035012 (2009) doi:10.1103/PhysRevD.79.035012 [arXiv:0809.0202 [hep-ph]].

[30] K. Hagiwara, S. Ishihara, J. Kamoshita and B. A. Kniehl, Eur. Phys. J. C 14, 457 (2000) doi:10.1007/s100520000366 hep-ph/0002043.

[31] D. Chang, W. Y. Keung and I. Phillips, Phys. Rev. D 48, 3225 (1993) doi:10.1103/PhysRevD.48.3225 hep-ph/9303226].

[32] S. S. Biswal and R. M. Godbole, Phys. Lett. B 680, 81 (2009) doi:10.1016/j.physletb.2009.08.014 arXiv:0906.5471 [hep-ph]].

[33] S. Dutta, K. Hagiwara and Y. Matsumoto, Phys. Rev. D 78, 115016 (2008) doi:10.1103/PhysRevD.78.115016 [arXiv:0808.0477 [hep-ph]].

[34] S. S. Biswal, R. M. Godbole, B. Mellado and S. Raychaudhuri, Phys. Rev. Lett. 109, 261801 (2012) doi:10.1103/PhysRevLett.109.261801 [arXiv:1203.6285 [hep-ph]].

[35] T. Han and Y. Li, Phys. Lett. B 683, 278 (2010) [arXiv:0911.2933 [hep-ph]].

[36] N. D. Christensen, T. Han and Y. Li, Phys. Lett. B 693, 28 (2010) [arXiv:1005.5393 [hep-ph]].

[37] C. Englert, D. Goncalves-Netto, K. Mawatari and T. Plehn, JHEP 1301, 148 (2013) doi:10.1007/JHEP01(2013)148 arXiv:1212.0843 [hep-ph]]. 
[38] S. S. Biswal, R. M. Godbole, R. K. Singh and D. Choudhury, Phys. Rev. D 73, 035001 (2006) [Phys. Rev. D 74, 039904 (2006)] doi:10.1103/PhysRevD.74.039904, 10.1103/PhysRevD.73.035001 [hep-ph/0509070].

[39] R. M. Godbole, D. J. Miller and M. M. Muhlleitner, JHEP 0712, 031 (2007) doi:10.1088/11266708/2007/12/031 [arXiv:0708.0458 [hep-ph]].

[40] B. Bhattacherjee, T. Modak, S. K. Patra and R. Sinha, arXiv:1503.08924 [hep-ph].

[41] M. Beneke, D. Boito and Y. M. Wang, JHEP 1411, 028 (2014) arXiv:1406.1361 [hep-ph]].

[42] R. M. Godbole, D. J. Miller, K. A. Mohan and C. D. White, JHEP 1504, 103 (2015) arXiv:1409.5449 [hep-ph]].

[43] S. Dawson, S. K. Gupta and G. Valencia, Phys. Rev. D 88, no. 3, 035008 (2013) arXiv:1304.3514 [hep-ph]].

[44] A. Kruse, A. S. Cornell, M. Kumar, B. Mellado and X. Ruan, Phys. Rev. D 91, no. 5, 053009 (2015) doi:10.1103/PhysRevD.91.053009 [arXiv:1412.4710 [hep-ph]].

[45] R. Rattazzi, Z. Phys. C 40, 605 (1988). doi:10.1007/BF01560232

[46] K. Hagiwara and M. L. Stong, Z. Phys. C 62, 99 (1994) doi:10.1007/BF01559529 hep-ph/9309248.

[47] G. J. Gounaris, F. M. Renard and N. D. Vlachos, Nucl. Phys. B 459, 51 (1996) doi:10.1016/0550-3213(95)00602-8 [hep-ph/9509316].

[48] P. Niezurawski, A. F. Zarnecki and M. Krawczyk, Acta Phys. Polon. B 36, 833 (2005) hep-ph/0410291.

[49] A. Skjold and P. Osland, Nucl. Phys. B 453, 3 (1995) doi:10.1016/0550-3213(95)00373-Z hep-ph/9502283.

[50] A. Skjold and P. Osland, Phys. Lett. B 311, 261 (1993) doi:10.1016/0370-2693(93)90565-Y hep-ph/9303294.

[51] A. Skjold and P. Osland, Phys. Lett. B 329, 305 (1994) doi:10.1016/0370-2693(94)90777-3 hep-ph/9402358.

[52] B. Grzadkowski and J. F. Gunion, Phys. Lett. B 350, 218 (1995) doi:10.1016/03702693(95)00369-V hep-ph/9501339.

[53] R. M. Godbole, Pramana 67, 835 (2006). doi:10.1007/s12043-006-0096-8

[54] B. Zhang, Y. P. Kuang, H. J. He and C. P. Yuan, Phys. Rev. D 67, 114024 (2003) doi:10.1103/PhysRevD.67.114024 [hep-ph/0303048].

[55] C. P. Buszello and P. Marquard, hep-ph/0603209.

[56] V. Hankele, G. Klamke and D. Zeppenfeld, hep-ph/0605117.

[57] W. Buchmuller and D. Wyler, Nucl. Phys. B 268, 621 (1986).

[58] B. Grzadkowski, M. Iskrzynski, M. Misiak and J. Rosiek, JHEP 1010, 085 (2010) arXiv:1008.4884 [hep-ph]]. 
[59] R. Contino, M. Ghezzi, C. Grojean, M. Muhlleitner and M. Spira, JHEP 1307, 035 (2013) doi:10.1007/JHEP07(2013)035 arXiv:1303.3876 [hep-ph]].

[60] C. N. Leung, S. T. Love and S. Rao, Z. Phys. C 31, 433 (1986). doi:10.1007/BF01588041

[61] K. Hagiwara, R. Szalapski and D. Zeppenfeld, Phys. Lett. B 318, 155 (1993) doi:10.1016/03702693(93)91799-S hep-ph/9308347].

[62] M. C. Gonzalez-Garcia, Int. J. Mod. Phys. A 14, 3121 (1999) hep-ph/9902321].

[63] Y. T. Chien, V. Cirigliano, W. Dekens, J. de Vries and E. Mereghetti, JHEP 1602, 011 (2016) [JHEP 1602, 011 (2016)] doi:10.1007/JHEP02(2016)011 [arXiv:1510.00725 [hep-ph]].

[64] V. Cirigliano, W. Dekens, J. de Vries and E. Mereghetti, arXiv:1603.03049 [hep-ph].

[65] S. Dwivedi, D. K. Ghosh, B. Mukhopadhyaya and A. Shivaji, Phys. Rev. D 92, no. 9, 095015 (2015) doi:10.1103/PhysRevD.92.095015 [arXiv:1505.05844 [hep-ph]].

[66] D. Choudhury, R. Islam and A. Kundu, Phys. Rev. D 88, no. 1, 013014 (2013) doi:10.1103/PhysRevD.88.013014 [arXiv:1212.4652 [hep-ph]].

[67] M. Dahiya, S. Dutta and R. Islam, Phys. Rev. D 93, no. 5, 055013 (2016) doi:10.1103/PhysRevD.93.055013 arXiv:1311.4523 [hep-ph]].

[68] G. Valencia, In *Boulder 1994, Proceedings, CP violation and the limits of the standard model* 235-269, and Iowa State U. Ames - AMES-HET-94-12 (94,rec.Dec.) $35 \mathrm{p}$ hep-ph/9411441.

[69] J. Alwall, R. Frederix, S. Frixione, V. Hirschi, F. Maltoni, O. Mattelaer, H.-S. Shao and T. Stelzer et al., JHEP 1407, 079 (2014) arXiv:1405.0301 [hep-ph]].

[70] N. D. Christensen and C. Duhr, Comput. Phys. Commun. 180, 1614 (2009) arXiv:0806.4194 [hep-ph]].

[71] R. D. Ball et al., Nucl. Phys. B 867, 244 (2013) doi:10.1016/j.nuclphysb.2012.10.003 arXiv:1207.1303 [hep-ph]].

[72] T. Sjostrand, S. Mrenna and P. Z. Skands, JHEP 0605, 026 (2006) doi:10.1088/1126$6708 / 2006 / 05 / 026$ [hep-ph/0603175].

[73] J. de Favereau et al. [DELPHES 3 Collaboration], JHEP 1402, 057 (2014) doi:10.1007/JHEP02(2014)057 arXiv:1307.6346 [hep-ex]].

[74] https://root.cern.ch/

[75] S. Banerjee, T. Mandal, B. Mellado and B. Mukhopadhyaya, JHEP 1509, 057 (2015) arXiv:1505.00226 [hep-ph]]. 